\title{
Temperatura e enriquecimento ambiental sobre o bem-estar de coelhos em crescimento
}

\author{
Temperature and environmental enrichment on the welfare of growing rabbits
}

\author{
Estela Valéria Siloto ${ }^{I}$ Cynthia Pieri Zeferino ${ }^{I}$ Ana Silvia Alves Meira Tavares Moura ${ }^{\mathrm{II} *}$ \\ Simone Fernandes ${ }^{\text {II }}$ José Roberto Sartori ${ }^{\text {III }}$ Edson Ramos de Siqueira ${ }^{I I}$
}

\section{RESUMO}

O efeito da temperatura e o efeito do enriquecimento do piso de gaiola sobre o bem-estar de coelhos em crescimento foram avaliados. Quarenta e oito coelhos do grupo genético Botucatu, desmamados aos 35 dias de idade, foram alojados em Câmara Climática, quatro por gaiola (1,00 x 0,60 x 0,40m), em 12 gaiolas que tinham (ou não) metade do piso coberto com cama de palha. As gaiolas foram instaladas metade em sala com temperatura ambiente e metade em sala resfriada. Os animais tiveram livre acesso à ração balanceada e à água nos dois tipos de piso. Para se avaliar o bem-estar, foram realizadas cinco observações de 24 horas cada, uma por semana, sendo registradas as freqüencias dos comportamentos: lúdico, estereotipado, exploratório, cuidados corporais e interação e comparadas entre gaiolas enriquecidas ou não nas duas salas. A média de temperatura e a média de umidade relativa do ar foram: $23,6^{\circ} \mathrm{C}$ e $78,7 \%$ na sala natural e $20,6^{\circ} \mathrm{C}$ e $71,0 \%$ na sala resfriada. O comportamento lúdico foi mais freqüente nas gaiolas enriquecidas (7,6 vs 4,3\% sala natural e 7,8 vs $3,8 \%$ sala resfriada, $P<0,01)$ e as estereotipias foram mais freqüentes nas gaiolas sem enriquecimento $(4,4$ vs. $2,7 \%$ sala natural e 2,1 vs $1,1 \%$ sala resfriada, $P>0,01$ ). Coelhos mantidos em temperatura natural preferiram a grade à cama de palha (77,9 vs. 22,1\%, P>0,01), enquanto na sala resfriada eles não mostraram preferência em relação ao piso (45,9 vs $54,1 \%, P=0,41)$. Em sala resfriada, o enriquecimento com palha favoreceu o bem-estar animal.

Palavras-chave: comportamento, estresse, Oryctolagus cuniculus, piso da gaiola, temperatura ambiente.

\section{ABSTRACT}

The effects of temperature and cage floor enrichment on the welfare of growing rabbits were evaluated. Forty-eight rabbits from the Botucatu genetic group, weaned at 35 days of age were housed in an Environmental Chamber, four per cage $(1.00 \times 0.60 \times 0.40 \mathrm{~m})$, in 12 cages that had (or not) half of the floor covered with litter straw. The cages were housed half in a room temperature chamber and half in a refrigerated chamber. Animals had free access to a balanced feed and water in the two types of floor. In order to evaluate welfare, five 24-hour observations were carried out, one per week, to register the frequencies of the following behaviors: ludic, stereotypic, exploratory, self-grooming and interactions, and to compare them between enriched and non-enriched cages from the two chambers. The average temperatures and relative humidities were $23.6^{\circ} \mathrm{C}$ and $78.7 \%$ in the room temperature chamber and $20.6^{\circ} \mathrm{C}$ and $71.0 \%$ in the refrigerated chamber. The ludic behavior was more frequent in the enriched cages (7.6 vs. $4.3 \%$ Room Temperature Chamber and 7.8 vs. $3.8 \%$ Refrigerated Chamber, $P<0.01)$ and the stereotypies in the non-enriched cages (4.4 vs. 2.7\% Room Temperature Chamber and 2.1 vs $1.1 \%$ Refrigerated Chamber, $P<0.01)$. Rabbits kept at room temperature preferred the wire net floor over the litter straw (77.9 vs. $22.1 \%, P<0.01)$, whereas in the refrigerated chamber they did not show preference regarding floor types (45.9 vs. $54.1 \%, P=0.41$ ). In the refrigerated chamber, cage floor enrichment favored animal welfare.

Key words: ambient temperature, behavior, cage floor, Oryctolagus cuniculus, stress.

IPrograma de Pós-graduação em Zootecnia, Faculdade de Medicina Veterinária e Zootecnia (FMVZ), Universidade Estadual Paulista (UNESP), Botucatu, SP, Brasil.

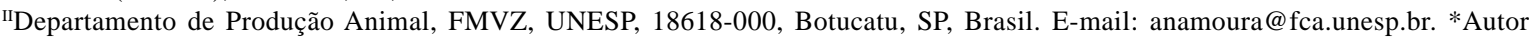
para correspondência.

IIIDepartamento de Melhoramento e Nutrição Animal, FMVZ, UNESP, Botucatu, SP, Brasil. 


\section{INTRODUÇÃO}

Nos últimos anos, a análise do bem-estar na criação de coelhos tem merecido atenção e muitas pesquisas visam avaliar alternativas nos sistemas de alojamento (HOY, 2006; VERGA et al., 2007). Os consumidores em todo o mundo estão aumentando suas exigências com relação às garantias de bem-estar dos animais, criando expectativas de como os animais devem ser tratados e como os alimentos devem ser produzidos (EFSA, 2005).

A interação entre fatores genéticos, nutricionais e principalmente ambientais é fundamental na determinação de uma eficiente produção de coelhos. Fatores ambientais representados pela temperatura, pela umidade e pela ventilação são os que mais influenciam o bem-estar e, conseqüentemente, a saúde, a reprodução e a produção animal.

A temperatura ideal para coelhos, a partir do desmame, varia de 15 a $20^{\circ} \mathrm{C}$ e a umidade relativa varia de 60 a 70\% (LEBAS et al., 1996), sendo assim, a simples proteção da radiação solar, seja por meio de sombreamento natural ou artificial, se torna indispensável para o fornecimento de um ambiente favorável aos animais. Ambientes com menor condição de renovação de ar e com instalação mais fechada geram piores resultados de produção, sendo, portanto, indispensável que as instalações forneçam condições ambientais próximas à zona de conforto térmico para favorecer o bem-estar do animal, evitando estresses e potencializando a produção.

O tipo de piso utilizado nas instalações é de fundamental importância, pois está diretamente relacionado à sanidade animal. A temperatura e a umidade inadequadas propiciam a proliferação de microorganismos patogênicos, favorecendo o aparecimento de doenças, principalmente quando se utiliza cama. Esta pode ser constituída de quaisquer materiais secos, fibrosos e que não sejam prejudiciais aos animais, de alta disponibilidade e bom preço, devendo ser renovada freqüentemente. $\mathrm{O}$ piso deve ser de fácil limpeza e evitar o contato direto dos animais com suas fezes (BARBOSA et al., 1992).

O bem-estar do coelho depende, em grande parte, do espaço disponível; as gaiolas demasiadamente pequenas ou a lotação excessiva limitam os movimentos, impedindo os animais de determinadas manifestações naturais, com conseqüentes alterações de ordem higiênico-sanitária, de comportamento e produtiva (ARVEAUX, 1991). Portanto, este estudo teve como objetivo avaliar o efeito da temperatura ambiente e do enriquecimento no piso da gaiola sobre o comportamento e o bem-estar de coelhos da desmama até a idade de abate, aos 70 dias.

\section{MATERIAL E MÉTODOS}

O experimento foi realizado na Câmara Bioclimática da Faculdade de Medicina Veterinária e Zootecnia (FMVZ) da Universidade Estadual Paulista (UNESP), Campus de Botucatu. Foram utilizados 48 coelhos desmamados pertencentes ao grupo genético Botucatu, que é uma linhagem sintética originada de híbridos Norfolk 2000 (MOURA et al., 2001). As mães foram alojadas em gaiolas metálicas elevadas de arame galvanizado, no galpão maternidade que tem orientação leste-oeste, é aberto e possui cortinas plásticas reguláveis nas laterais. Aos 35 dias, foi realizada a desmama e os coelhos receberam tatuagem na orelha direita para a identificação individual.

Os coelhos desmamados foram alojados ao acaso, quatro animais por gaiola, em duas salas na Câmara Climática, até atingirem a idade de setenta dias. A densidade de alojamento adotada neste estudo foi baixa, quatro animais por gaiola de dimensões $1,00 \mathrm{x}$ $0,60 \times 0,40 \mathrm{~m}$, ou seja, $1000 \mathrm{~cm}^{2}$ animal ${ }^{-1}$. Cada sala abrigou seis gaiolas, sendo três com enriquecimento do piso (piso misto) e três somente com piso de grade de arame. As gaiolas de arame galvanizado foram instaladas em paralelo e equipadas com dois bebedouros e dois comedouros posicionados em suas extremidades. As gaiolas com piso misto continham metade da superfície coberta com cama de palha, permitindo livre acesso dos animais aos dois diferentes tipos de piso.

A ração fornecida foi peletizada, de composição balanceada (2500kcal kg-1 ED; 16,0\% PB; 16,7\% FDA), conforme exigências nutricionais descritas por DE BLAS \& MATEOS (1998), e foi produzida na fábrica de rações da FMVZ. A ração foi fornecida à vontade durante todo o período experimental, assim como a água.

Os animais foram submetidos a duas condições diferentes de temperatura: uma sala foi mantida à temperatura ambiente (sala natural) e a outra foi resfriada por meio da instalação de um aparelho de ar condicionado para proporcionar temperatura próxima à termoneutralidade (sala resfriada). A temperatura máxima e a mínima e a umidade relativa do ar foram monitoradas diariamente às $9 \mathrm{~h}, 14 \mathrm{~h}$ e $21 \mathrm{~h}$ durante todo o período experimental. Nos cinco dias de observação, a temperatura e a umidade foram registradas a cada duas horas, obtendo-se a média do dia. Nos cálculos da temperatura média e da umidade relativa do ar para o período experimental, foram utilizadas as fórmulas propostas pelo Inmet (MAPA), segundo MULLER (1989): $\mathrm{Tm}_{\text {ed }}=\left(\mathrm{T}_{9 \mathrm{~h}}+\mathrm{T}_{\text {máx }}+\mathrm{T}_{\text {mín }}+2 . \mathrm{T}_{21 \mathrm{~h}}\right) / 5$ em que: $\mathrm{T}_{\text {med }}=$ temperatura média do ar; $\mathrm{T}_{9 \mathrm{~h}}=$ temperatura do ar registrada às $9 \mathrm{~h} ; \mathrm{T}_{\text {máx }}=$ temperatura máxima do ar 
registrada às $21 \mathrm{~h} ; \mathrm{T}_{\min }=$ temperatura mínima do ar registrada às $21 \mathrm{~h} ; \mathrm{T}_{21 \mathrm{~h}}^{\text {min }}=$ temperatura mínima do ar registrada às $21 \mathrm{~h}$.

$$
\mathrm{U}=\left(\mathrm{U}_{9 \mathrm{~h}}+\mathrm{U}_{14 \mathrm{~h}}+2 . \mathrm{U}_{21 \mathrm{~h}}\right) / 4
$$

em que: $U$ = umidade relativa média diária do ar; $U_{9 h}=$ umidade relativa do ar registrada às $9 \mathrm{~h}$; $U_{14 h}=$ umidade relativa do ar registrada às $14 \mathrm{~h} ; \mathrm{U}_{21 \mathrm{~h}}=$ umidade relativa do ar registrada às $21 \mathrm{~h}$.

Os animais foram distribuídos em um delineamento inteiramente casualizado, com arranjo fatorial 2 x 2 (duas condições térmicas e dois tipos de piso). A preferência pelo tipo de piso e o estado de bem-estar dos animais foram avaliados por meio de observações realizadas uma vez por semana, com duração de 24 horas contínuas, num total de 120 horas de observação distribuídas no período de cinco semanas.

As observações foram efetuadas de duas formas: 1. Sistema de varredura - a cada cinco minutos foram contados quantos animais estavam sobre a grade ou sobre a cama nas gaiolas com enriquecimento do piso, não sendo considerados os animais que estavam comendo e bebendo; e 2. Registro dos comportamentos - lúdico, estereotipado, exploratório, cuidados corporais e interação dos indivíduos nas gaiolas enriquecidas ou não, em todas as suas ocorrências.

A freqüência de permanência sobre a grade ou sobre a cama nas gaiolas enriquecidas foi analisada por meio do teste de qui-quadrado. Os efeitos da temperatura da sala, do dia e da hora de observação sobre a incidência de animais na grade ou na cama foram investigados utilizando-se análise de variância. O modelo incluiu os três efeitos fixos e as interações.
Para a avaliação da preferência e do bem-estar em relação ao tipo de piso, foram utilizadas a presença do comportamento lúdico e a ausência do estereotipado, além da permanência em cada piso.

\section{RESULTADOS E DISCUSSÃO}

As médias de temperatura e umidade relativa do ar obtidas nos cinco dias de observação comportamental dos animais foram: $23,6^{\circ} \mathrm{C}$ e $78,7 \%$ na sala natural e $20,6^{\circ} \mathrm{C}$ e $71,0 \%$ na sala resfriada. Deve-se ressaltar que no segundo dia de observação houve queda de energia elétrica no período das $3 \mathrm{~h}$ às $9 \mathrm{~h} 30 \mathrm{~min}$ e no último dia de observação, das 12h35min às 16h, provocando elevação da temperatura e umidade na sala resfriada.

A permanência dos animais sobre a grade ou sobre a cama nas gaiolas enriquecidas diferiu entre as salas (Figura 1). Na sala natural, a maior parte dos animais permaneceu na grade $(\mathrm{P}<0,01)$. Na sala resfriada, não houve diferença entre freqüência na grade ou na cama. A preferência pelo tipo de piso da gaiola e a densidade populacional foi analisada por OROVA et al. (2004), comprovando que coelhos mantidos entre $16 \mathrm{e}$ $18^{\circ} \mathrm{C}$ na Hungria onde o estudo foi conduzido, permaneceram mais tempo em piso de arame do que em piso forrado. Os pesquisadores concluíram também que a densidade de alojamento tem influência significativa na escolha entre o tipo de piso, sendo a preferência pelo piso com palha maior (18\%) em gaiolas que continham 8 animais $\mathrm{m}^{-2}$ e menor (14\%) nas gaiolas que continham 12 e 16 animais $\mathrm{m}^{-2}$.

Houve efeito da interação temperatura da sala x dia x hora sobre a incidência de animais $(\mathrm{P}<0,01)$

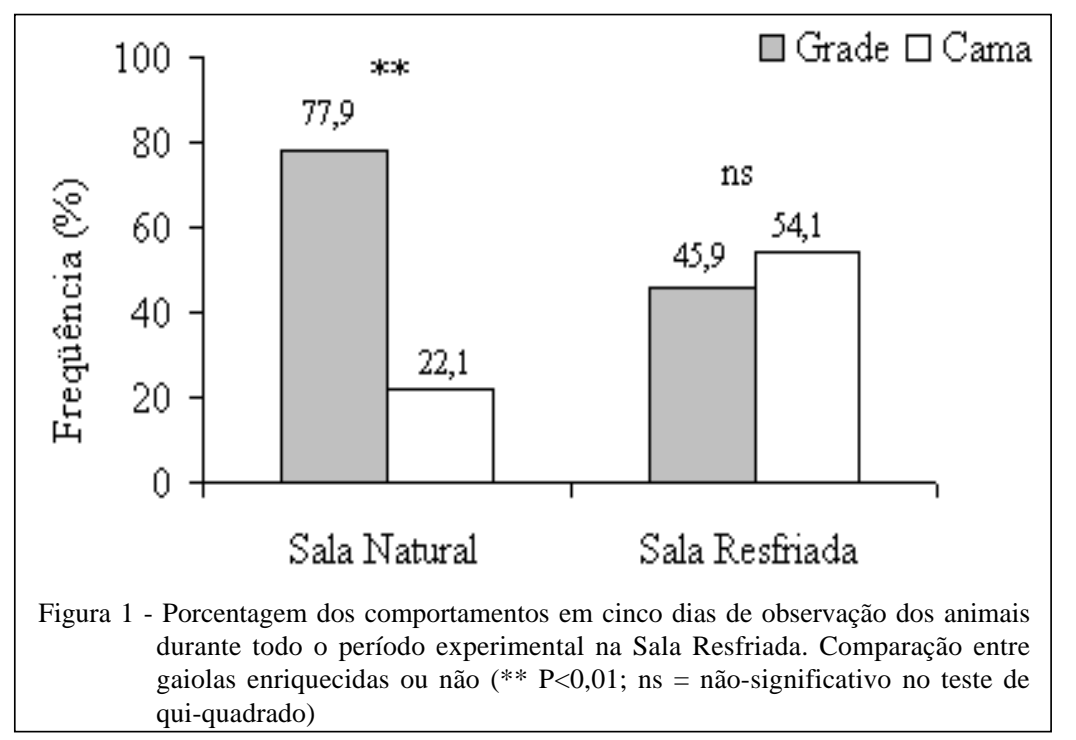

Ciência Rural, v.39, n.2, mar-abr, 2009. 
na grade ou na cama, nas gaiolas enriquecidas. Na sala natural, houve maior incidência de animais aos 38, 52 e 59 dias de idade sobre a cama das 2 às 8 h e no restante do tempo houve preferência pela grade. Essa preferência pode estar relacionada à temperatura ambiente, pois a permanência na cama foi maior durante a noite, quando a temperatura estava mais baixa. Já durante o dia, quando a temperatura se elevava, a grade permitiu maior troca de calor com o ambiente, contribuindo com o conforto térmico.

Na sala resfriada, ocorreu o oposto. A permanência sobre a grade aos 38 e 45 dias foi maior durante a noite, das 18 às 6h. Aos 52 dias, houve uma diminuição da freqüência na grade e um aumento na cama durante esse período, praticamente igualandoas. Durante o dia, em todas as fases de vida dos animais, a permanência na cama foi superior das 6 às 16h. Nessa sala, em que não houve grandes alterações na temperatura entre dia e noite, a preferência dos animais jovens pela grade pode ter sido influenciada pela maternidade, pois os animais eram mantidos com suas mães em gaiolas convencionais. Nesse caso, a preferência pela grade coincidiu com o período de maior atividade dos animais. Em contraste, praticamente em todas as idades, houve preferência pela cama no período de menor atividade (entre 8h e 16h). Aos 59 dias, a permanência na cama aumentou no período noturno, mantendo-se ainda inferior à grade.

Animais com cinco a seis semanas de idade, mantidos entre 16 e $18^{\circ} \mathrm{C}$, permaneceram $78 \%$ do tempo sobre piso de arame e $22 \%$ sobre piso forrado com palha. Já quando estavam com nove a 10 semanas, esta proporção foi de $81 \%$ sobre piso de arame e $19 \%$ sobre piso forrado (OROVA et al., 2004)
Para análise dos registros de eventos comportamentais, foram consideradas todas as gaiolas, com e sem enriquecimento, e foi feita a soma das ocorrências nas cinco observações realizadas. Os coelhos apresentam menor atividade das $6 \mathrm{~h}$ às $18 \mathrm{~h}$. Esse dado se assemelha com o encontrado no trabalho de GUNN \& MORTON (1995), em que o comportamento sleep, relacionado com estado de dormência dos animais, se manteve elevado entre 6h e 16h, sofrendo um imediato aumento quando se iniciou o período de luz do dia e durando, em média, 9h dia $^{-1}$.

A brincadeira é um comportamento facilmente detectado, mas de difícil definição (BEKOFF \& ALLEN, 1998), pois incorpora muitos componentes dos padrões comportamentais dos adultos, podendo ser exagerada, repetitiva e variada (WALTERS, 1987). Há três tipos de brincadeiras comumente aceitos: brincadeira com objetos, na qual o animal manipula um objeto de maneira repetitiva (lúdica), brincadeira locomotora, em que o animal pula e corre sozinho e brincadeira social, que envolve mais de um indivíduo (WALTERS, 1987; BURGHART, 1998). O comportamento lúdico no presente estudo foi observado quando os animais pulavam, corriam e interagiam uns com os outros por meio de saltos e movimentos que refletiam um estado de brincadeira. Os coelhos das gaiolas com enriquecimento ambiental em ambas as salas apresentaram maior frequência de comportamento lúdico (Figuras 2 e 3). VERGA et al. (2004) também observaram em seu experimento, comparando coelhos mantidos em gaiolas enriquecidas com palha e em gaiolas convencionais, uma diferença de comportamentos lúdicos quando havia enriquecimento.

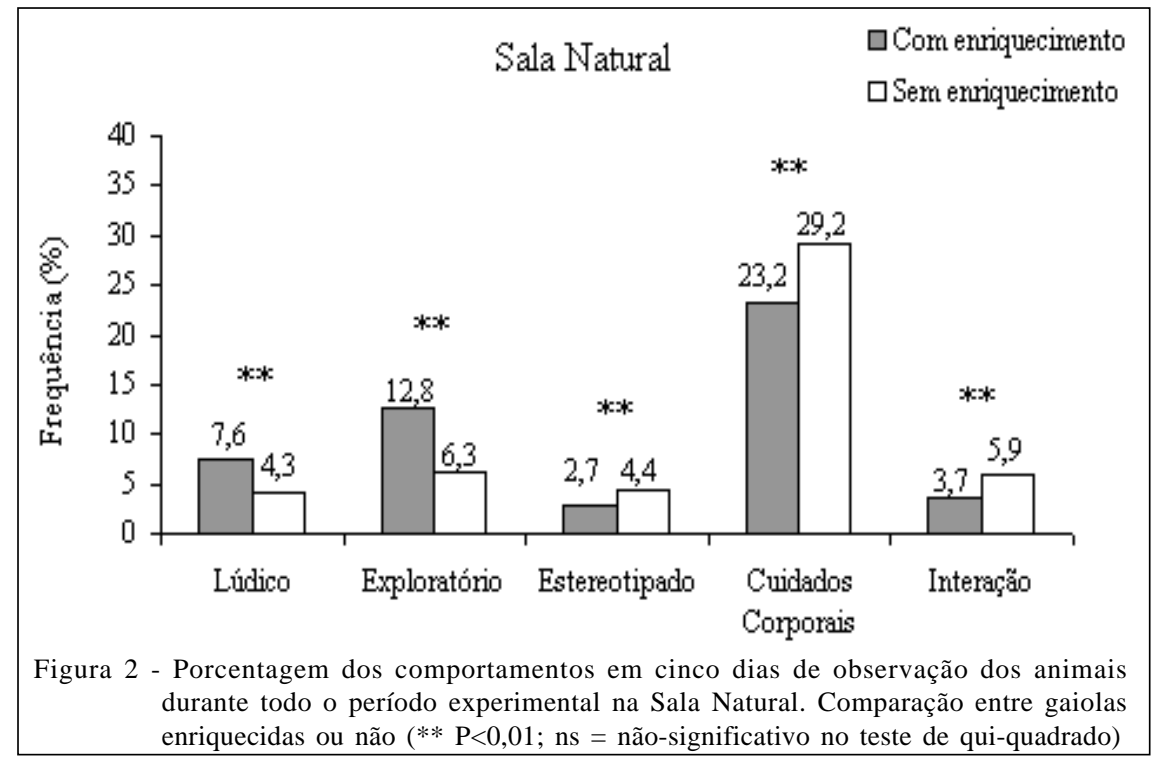

Ciência Rural, v.39, n.2, mar-abr, 2009. 


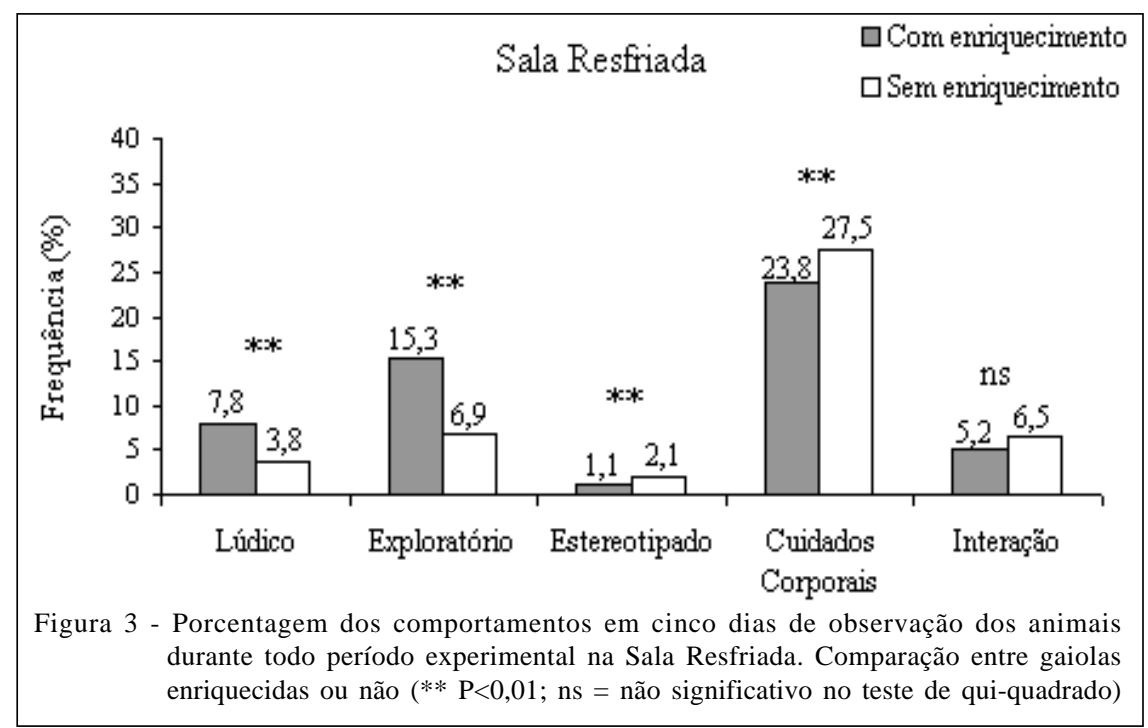

Já o comportamento estereotipado, manifestado geralmente quando roíam a grade ou o bebedouro, conforme indicado por TROCINO \& XICCATO (2006), se sobressaiu nas gaiolas sem enriquecimento da sala natural (Figura 2). Poder-se-ia aventar a hipótese deste não ser um comportamento estereotipado e sim exploratório, porém, o tempo e a insistência da mordedura foram esclarecedores em relação a essa afirmação. A diferenciação entre comportamento estereotipado e exploratório, nesse caso, efetuou-se pelo tempo e pela atitude dos animais em relação à ação de mordedura da grade. Na sala resfriada, a frequiência de estereotipias também foi menor nas gaiolas com enriquecimento (Figura 3). GUNN \& MORTON (1995) observaram coelhos em gaiolas de laboratório (individuais) e a presença de estereotipias foi maior durante a noite, por volta das 3h, quando os animais estavam em alerta, mas sem desenvolver muitas atividades, semelhante ao que ocorreu no presente estudo. Essas manifestações de estereotipias são consideradas desvios de comportamento, indicando baixa adaptação ao ambiente.

O comportamento exploratório, ou interação com o ambiente, foi observado quando os animais roíam a palha, cheiravam o ar e interagiam com o observador. Esse comportamento foi observado com maior freqüência nas gaiolas com enriquecimento de ambas as salas (Figuras 2 e 3 ). As gaiolas sem enriquecimento tiveram freqüências semelhantes. Esse resultado concorda com o obtido por VERGA et al. (2004), os quais afirmaram que coelhos mantidos em gaiolas enriquecidas apresentaram maior freqüência de comportamento exploratório e agitação, permanecendo menos tempo deitados ou em ócio que coelhos mantidos em gaiolas sem enriquecimento. $\mathrm{O}$ comportamento de fêmeas colocadas em gaiolas com palha foi verificado por LÓPEZ et al. (2004), os quais observaram que mais de $70 \%$ dos coelhos ocuparam as gaiolas que continham palha imediatamente após a introdução da mesma, sendo o interesse menor após a primeira hora da disposição do material. A freqüência de cuidados corporais foi semelhante nas gaiolas enriquecidas ou não da sala resfriada (Figura 3), mas foi mais elevada nas gaiolas sem enriquecimento da sala natural (Figura 2). HANSEN \& BERTHELSEN (2000) afirmaram que animais mantidos em gaiolas convencionais enriquecidas passaram boa parte de seu tempo realizando cuidados corporais. Estudos citados no trabalho desses pesquisadores revelam que coelhos selvagens realizam esse comportamento em apenas $2 \%$ do período de 24 horas. Quando os cuidados corporais se manifestam freqüentemente, mas em períodos de curta duração, é possível que reflitam uma situação de estereotipia, pois o animal não os realiza com o propósito de higiene corporal. Esse comportamento, quando excessivo, mesmo em gaiolas enriquecidas, pode indicar falta de estímulo pelo ambiente ou, como GUNN \& MORTON (1995) sugeriram, pode ser um estado de privação social.

A interação social, considerada quando um coelho realizava cuidados corporais em outro, apresentou um pico nas gaiolas sem enriquecimento da sala natural entre $2 \mathrm{~h}$ e $4 \mathrm{~h}$. Essa freqüência foi menor nas gaiolas com enriquecimento ambiental de ambas as salas (Figuras 2 e 3 ).

\section{CONCLUSÕES}

Coelhos mantidos em sala com temperatura superior a $20^{\circ} \mathrm{C}$ tenderam a permanecer maior parte do 
tempo sobre o piso de arame. Em sala resfriada, os comportamentos apresentados indicaram que o uso do piso enriquecido favoreceu o bem-estar animal. O enriquecimento ambiental é um meio de satisfazer as necessidades comportamentais de coelhos mantidos em gaiolas.

\section{COMITÊ DE ÉTICA E BIOSSEGURANÇA}

Este trabalho foi aprovado pela Câmara de Ética em Experimentação Animal da Faculdade de Medicina Veterinária e Zootecnia em 23 de setembro de 2005 (protocolo no 072/ 2005 - CEEA).

\section{AGRADECIMENTOS}

Os autores agradecem aos seguintes colaboradores: Irene Francisca de Arruda, Brenda B. L. Medeiros, Gisele F. Túlio, Fábio T. F. Muino, Fabrizia S. Otani, Vitor H. C. Novaes, Juliana S. Kanayama, Mariana Trevisanuto e Mariana de Carvalho, pelo auxílio na observação dos animais. Cynthia Pieri Zeferino recebeu bolsa de Iniciação Científica da FAPESP para desenvolver este projeto.

\section{REFERÊNCIAS}

ARVEAUX, P. Densidad en jaulas de engorde. Cunicultura, v.16, n.90, p.107, 1991.

BARBOSA, O.R. et al. Desempenho de coelhos da raça Nova Zelândia Branco, criados em diferentes tipos de instalações, durante as estações do verão e do inverno. 1. Temperatura corporal, freqüência respiratória, consumo de ração, ganho de peso e conversão alimentar. Revista Brasileira de Zootecnia, v.21 n.5, p.779-786, 1992.

BEKOFF, M.; ALLEN, C. Intentional communication and social play: how and why animals negotiate and agree to play. In: BEKOFF, M.; BYERS, J.A. Animal play: evolutionary, comparative, and ecological perspectives. Cambridge and New York: Cambridge University, 1998. Cap.5, p.97-114.

BURGHART, G.M. The evolutionary origins of play revised: lessons from turtles. In: BEKOFF, M.; BYERS, J.A. Animal play: evolutionary, comparative, and ecological perspectives. Cambridge and New York: Cambridge University, 1998. Cap.1, p.1-26.

DE BLAS, C.; MATEOS, G.G. Feed formulation. In: DE BLAS, C.; WISERMAN, J. The nutrition of the rabbit. Cambridge: CABI, 1998. Cap.13, p.241-253.

EFSA (European Food and Safety Authority). The impact of the current housing and husbandry systems on the health and welfare of farmed domestic rabbits. EFSA Journal, v.267, p.1-31, 2005.
GUNN, D.; MORTON, D.B. Inventary of the behaviour of New Zealand White rabbits in laboratory cages. Applied Animal Behaviour Science, v.45, n.2, p.277-292, 1995.

HANSEN, L.T.; BERTHELSEN, H. The effect of environmental enrichment on the behaviour of caged rabbits (Oryctolagus cuniculus). Applied Animal Behaviour Science, v.68, n.2, p.163-178, 2000.

HOY S. Housing of rabbits in conformity with animal welfare and protection criteria. In: MAERTENS, L.; COUDERT, P. Recent advances in rabbit sciences. Melle: COST e ILVO, 2006. Cap.2, p.69-130.

LEBAS, F. et al. El conejo: cría y patología. Roma: FAO, 1996. 225p

LÓPEZ, M. et al. Evaluation of the use of straw as an entertainment in Gigante de España rabbit cages: the effect of the placing of the straw in cage on the behaviour.. In: WORLD RABBIT CONGRESS, 8., 2004, Puebla. Proceedings... Puebla: World Rabbit Science Association/ Universidad Politecnica de Valencia. 2004. p.1241-1246. CD-ROM.

MOURA, A.S.A.M.T. et al. Variance components and response to selection for reproductive, litter and growth traits through a multi-purpose index. World Rabbit Science, v.9, n.2, p.7786, 2001.

MULLER, P.B. Bioclimatologia aplicada aos animais domésticos. 3.ed. Porto Alegre: Sulina, 1989. 262p.

OROVA, Z. et al. Free choice of growing rabbits between deep litter and wire net floor in pens. In: WORLD RABBIT CONGRESS, 8., 2004, Puebla. Proceedings... Puebla: World Rabbit Science Association/ Universidad Politecnica de Valencia, 2004. p.1263-1265. CD-ROM.

TROCINO, A.; XICCATO, G. Animal welfare in reared rabbits: a review with emphasis on housing systems. World Rabbit Science, v.14, p.77-93, 2006.

VERGA, M. et al. Effect of housing, and environmental enrichment on performance and behaviour in fattening rabbits. In: WORLD RABBIT CONGRESS, 8., 2004, Puebla. Proceedings... Puebla: World Rabbit Science Association/ Universidad Politecnica de Valencia, 2004. p.1283-1289. CDROM.

VERGA, M. et al. Effects of husbandry and management systems on physiology and behaviour of farmed and laboratory rabbits. Hormones and Behavior, v.52, p.122-129, 2007.

WALTERS, J. Transition to adulthood. In SMUTS, B. et al. Primate societies. Chicago: University of Chicago, 1987. p.358-369. 